\title{
Food Bolus Impaction in the Era of Increased EoE Recognition: Push and Pull, Biopsy and Dilate Before It Is Too Late
}

\author{
Ajay Sharma ${ }^{1} \cdot$ Hamish Philpott ${ }^{2}$ \\ Published online: 12 April 2018 \\ (c) Springer Science+Business Media, LLC, part of Springer Nature 2018
}

Food bolus impaction of the esophagus (FBE), a common gastroenterological emergency, has been increasingly recognized as a complication of eosinophilic esophagitis (EoE) [1, 2]. The initial management of food bolus impaction is to first and foremost safely expedite relief of obstruction in order to prevent aspiration of gastric contents and esophageal perforation [3]. The second but similarly important priority is to consider the underlying cause in order to inform future management. It is in this latter case where clinical practice is arguably most divergent, and where consensus and future guidelines are also needed. Failure to commence appropriate treatment may not only lead to recurrent food bolus impaction, but also to the fibrostenosing form of $\operatorname{EoE}[4,5]$. The article by Hiremath et al. published in this issue of Digestive Diseases and Sciences [6] emphasizes the variability of the clinical management of food bolus impaction, discusses the significant divergence from "ideal" practice by summarizing the results of a 23 question survey administered to $>400$ adult and pediatric gastroenterologists (1:1) practicing in a variety of clinical settings including major academic facilities and privately run facilities, and speculates that failure to either correctly diagnose and/or appropriately treat EoE is commonplace [6].

In dealing with acute relief of obstruction, a range of devices were used, perhaps reflecting a preference by some practitioners, in particular pediatric gastroenterologists, to retrieve rather than push the bolus into the stomach. Indeed management guidelines are not uniform, with the American Society of Gastrointestinal Endoscopy (ASGE) cautioning against the "push" technique, while recent European

Hamish Philpott

lachlanphilpott2003@yahoo.com.au

1 Department of Paediatric Gastroenterology, Joondalup Health Campus, Joondalup, WA, Australia

2 Department of Gastroenterology, Northern Adelaide Local Health Network (NAHLN), University of Adelaide, Adelaide, SA, Australia guidelines recommend advancing the bolus into the stomach as a first-line safe, effective strategy [7, 8]. Interestingly, 28\% of respondents reported the availability of the recently tested and promising suction cap in order to facilitate retrieval [9]. Dilation of the esophagus following bolus clearance was reported as a preferred treatment by $\sim 50 \%$ of clinicians, foreseeably reflecting an easing of concerns regarding perforation in EoE, given recent reassuring data to this end [8].

With resolution of FBE, consideration of the underlying etiology should inform commencement of treatment. In the era of increased EoE recognition, biopsies have been advocated at the time of food bolus removal $[2,8]$. Of concern, biopsies were obtained in all FBE patients by only $\sim 1 / 3$ of clinicians, representing missed opportunities, broadly reflective of actual practice reported elsewhere [2]. Similarly, high-dose proton pump inhibitor (PPI) therapy was prescribed routinely by $<50 \%$ of respondents. Hiremath et al. compared this to a proposed "ideal" of best practice which quite logically and reasonably includes both biopsy and commencement of PPI therapy.

The methodology used by Hiremath et al. has significant shortcomings, most importantly being that data are theoretical rather than actual and responses are subject to significant bias. Notwithstanding, the real strength of such widespread data acquisition is that useful themes can and do emerge, including an estimation of the number of potential "missed" cases of EoE as a result of management divergent from best practice, which is estimated at 10,000 annually in the USA. Methodological flaws aside, the message is clear: Guidelines are needed, and patients with EoE will have their management compromised until these are instituted by pediatric and adult gastroenterologists en masse.

The management algorithm proposed by Hiremath et al. namely that biopsies be performed following relief of food bolus impaction, that PPI therapy is commenced, that repeat endoscopy is performed, and that a clinic appointment is scheduled, is logical and reasonable. Resource 


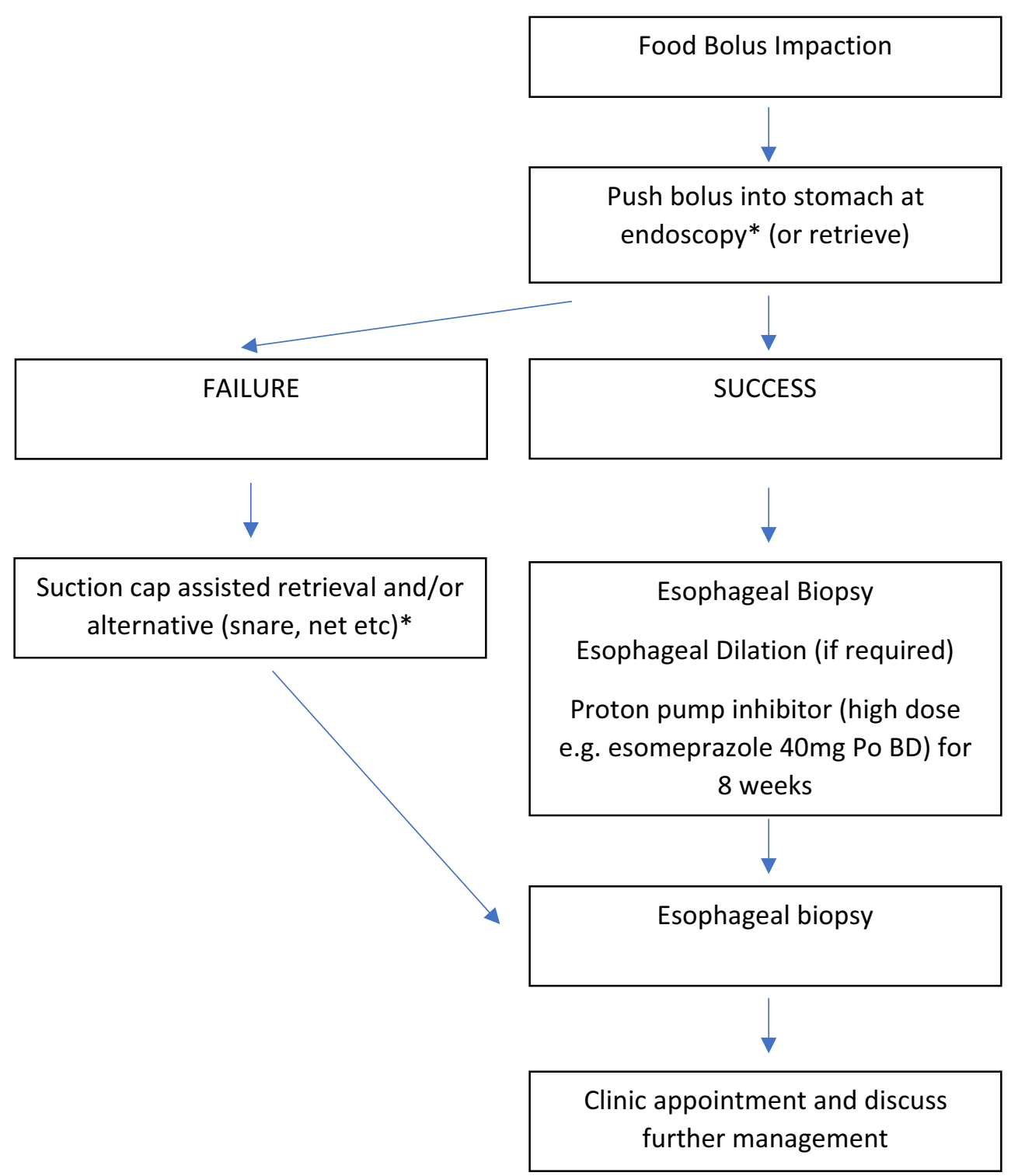

Fig. 1 Proposed ideal management of esophageal food impaction secondary to eosinophilic esophagitis. *Either advancing the food bolus into the stomach or retrieving the bolus is reasonable initial techniques, and individual clinician preference may dictate the approach

constraints aside, there is no major downside to these recommendations. That some patients may have food impaction despite PPI ("repeat offenders") of course requires separate consideration, aided by the use of alternative treatments such as topical corticosteroids and elimination diets. One indeed could go further, in specifying that a "push" technique should be used initially, that a suction cap should be used if this fails, and that dilation should be performed following relief from food impaction (Fig. 1). In the era of increased EoE awareness, push or pull, dilate, biopsy and PPIs along with rigorous follow-up are essential components of an ideal management strategy.

\section{References}

1. Sperry SL, Crockett SD, Miller CB, Shaheen NJ, Dellon ES. Esophageal foreign-body impactions: epidemiology, time trends, and the impact of the increasing prevalence of eosinophilic esophagitis. Gastrointest Endosc. 2011;74:985-991.

2. Philpott HL, Nandurkar S, Thien F, et al. Seasonal recurrence of food bolus obstruction in eosinophilic esophagitis. Intern Med J. 2015;45:939-943.

3. Runge TM, Eluri S, Cotton CC, et al. Causes and outcomes of esophageal perforation in eosinophilic esophagitis. J Clin Gastroenterol. 2016;111:206-213.

4. Runge TM, Eluri S, Cotton CC, et al. Outcomes of esophageal dilation in eosinophilic esophagitis: safety, efficacy, and persistence of the fibrostenotic phenotype. Am J Gastroenterol. 2016;111:206-213. 
5. Philpott H, Nandurkar S, Royce SG, Gibson PR. Letter: seasonality in eosinophilic oesophagitis and food bolus obstruction-What about recurrent episodes? Aliment Pharmacol Ther. 2015;42:936.

6. Hiremath, G, Vaezi, MF, Gupta, SK, Acra, S. Dellon, ES. Management of esophageal food impaction varies among gastroenterologists and affects identification of eosinophilic esophagitis. Dig Dis Sci. (Epub ahead of print). https://doi.org/10.1007/s1062 0-018-4972-0.

7. Birk M, Bauerfeind P, Deprez PH, et al. Removal of foreign bodies in the upper gastrointestinal tract in adults: European Society of
Gastrointestinal Endoscopy (ESGE) Clinical Guideline. Endoscopy. 2016;48:489-496.

8. Committee ASoP, Ikenberry SO, Jue TL, et al. Management of ingested foreign bodies and food impactions. Gastrointest Endosc. 2011;73:1085-1091.

9. Ooi M, Young EJ, Nguyen NQ. Effectiveness of a cap-assisted device in the endoscopic removal of food bolus obstruction from the esophagus. Gastrointest Endosc. 2018. https://doi. org/10.1016/j.gie.2017.12.022. 